\title{
Measurement of the Stimulated Carrier Lifetime in Semiconductor Optical Amplifiers by Four-Wave Mixing of Polarized ASE Noise
}

\author{
Guido Hunziker, Roberto Paiella, Student Member, IEEE, Kerry J. Vahala, Member, IEEE, and Uzi Koren
}

\begin{abstract}
We present a simple experiment aimed at measuring the stimulated carrier lifetime in semiconductor optical amplifiers (SOA's). The technique relies on polarization-resolved nearly degenerate four-wave mixing (FWM) of a laser source with an amplified spontaneous emission (ASE) noise source. The method can quickly characterize the bandwidth performance of active layers for application in a cross-gain or cross-phase wavelength converter.
\end{abstract}

Index Terms-Optical saturation, semiconductor lasers, stimulated emission, optical amplifiers, optical frequency conversion, optical mixers.

\section{INTRODUCTION}

$\mathbf{T}$ HE STIMULATED carrier lifetime of an optical amplifier is a key physical quantity in determining the amplifier gain, saturation power, as well as the onset and strength of nonlinear operation. Its small value in semiconductor optical amplifiers (SOA's), compared to Erbium doped fiber amplifiers (EDFA's), makes SOA's less attractive as linear amplifiers for $1.5-\mu \mathrm{m}$ wavelength systems. However, in nonlinear elements, a short stimulated lifetime is advantageous. For example, in wavelength converters exploiting cross-gain or cross-phase modulation, the stimulated carrier lifetime, $\tau_{s}$, is the limiting factor to bit-rate transparency, and much effort is being devoted to increase the corresponding corner frequency in the conversion efficiency [1]-[3]. In this letter, we present a novel technique for measuring $1 / \tau_{s}$ that uses the fact that this frequency is also the first corner frequency in the detuning dependence of the four-wave mixing (FWM) conversion efficiency [4], [5]. The approach does not require a network analyzer, tunable RF-sources, or tunable laser sources. The carrier lifetime corner frequency can be determined in just two RF-spectrum analyzer sweeps, using nothing but a continuouswave $(\mathrm{CW})$ laser (pump) and a polarized EDFA noise source ("probe").

\section{EXPERIMENT}

The measurement is based on polarization resolved, nearly degenerate FWM [4], [7] in an SOA where both TE and TM

Manuscript received January 22, 1997; revised April 4, 1997. This work was supported by ARPA under Grant DAAL 01-94-k-03430, by the National Science Foundation under Grant ECS 9412862, and by the NORTHROP Corporation.

G. Hunziker, R. Paiella, and K. J. Vahala are with the Department of Applied Physics, California Institute of Technology, Pasadena, CA 91125 USA.

U. Koren is with the Lucent Technologies, Holmdel, NJ 07733.

Publisher Item Identifier S 1041-1135(97)05023-4.

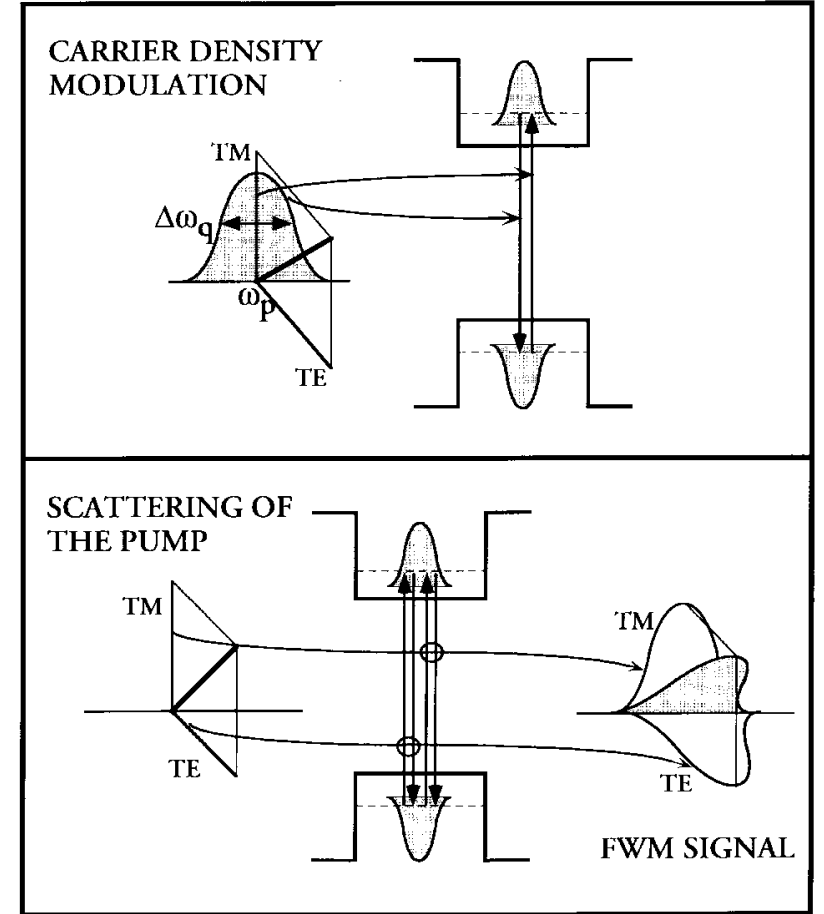

Fig. 1. Schematics of the FWM process showing the pump and probe polarizations used in this letter. In the upper panel, the generation of carrier density modulation by beating of the TM components of the input waves is shown. This occurs only in the tensile wells. In the lower panel, we show the scattering of each pump component into the corresponding FWM component. This occurs in both the tensile (TE and TM components) and in the compressively (TE only) strained wells. The probe is not a single frequency source but rather a continuum of frequencies within a $100-\mathrm{GHz}$ bandwidth. The modulation occurs at all of these frequencies, and the FWM signal is therefore also a continuum of frequencies.

polarized waves induce optical transitions. The generation of the FWM signal can be decomposed into two steps, as shown in Fig. 1: first, the input waves (pump and "probe") optically modulate the carrier density in the quantum wells and, second, the pump wave is scattered by the resulting gain and index modulation. Instead of using a single frequency probe laser beam, we use a broad-band ASE noise source from an EDFA, so that the frequency response can be characterized in one scan (this source is indicated as the gray broadband signal in Fig. 1). We will refer to this noise source as the "probe" despite the fact that it is not a single frequency laser. Notice that instead of using an EDFA, ASE from a secondary SOA could be used as a source. The polarization of this optical noise is set to be 


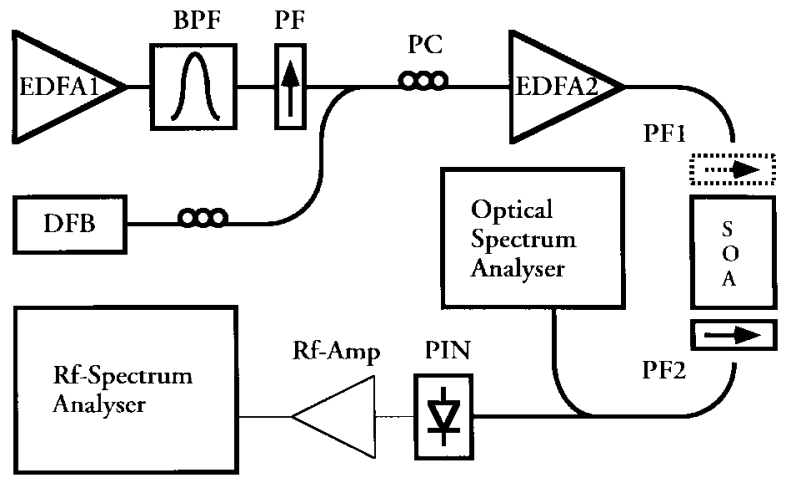

(a)

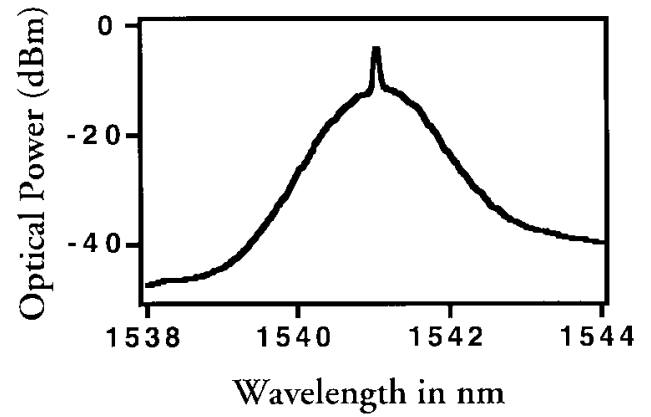

(b)

Fig. 2. (a) Experimental setup used to determine the stimulated carrier lifetime. The ASE from EDFA 1 is filtered in an optical bandpass filter (1.6-nm FWHM, labeled BPF in the figure) and then polarized in a fiber polarization filter (PF). This is then combined with the output of a DFB and amplified in EDFA 2. The polarization states are individually adjusted with mechanical polarization controllers (PC) and measured with a polarization filter (PF 1) before being coupled into the amplifier. PF 1 is subsequently removed and used at the output of the SOA (PF 2) to select either the TE or the TM emission for input to the photodetector (PIN). The RF-spectrum is amplified before being coupled into the spectrum analyzer. (b) Optical spectrum after the SOA, measured without any polarizers, showing the broad input noise source and the DFB line.

along TM by passing the EDFA emission though a polarization filter. As a result, the pump-"probe" beat note in the SOA is zero along the TE axis, and optically modulated carriers are only generated by beating of TM waves. However, the TMinduced carrier modulation will lead to a modulation of the gain and refractive index for both the TE and TM components of the pump, thereby generating the TE and TM polarized FWM signals. At the output of the device, we select the TE polarized waves, which, upon detection, will contain the beat note between the pump and the FWM-converted input noise. The frequency dependence of this signal is expected to reflect the first-order pole at the stimulated carrier recombination lifetime [4].

In the experiment, we launched a pump laser beam polarized at $45^{\circ}$ with respect to the growth (TM-) axis and a probe beam of TM-polarized ASE noise from an EDFA into the amplifier (see Fig. 2). The beating between the TM component of the pump (laser) and the "probe" (ASE noise) will generate a modulation of the carriers at all frequencies within the halfbandwidth of the ASE noise $(0.8 \mathrm{~nm}$ in this case, i.e., all frequencies from $0-100 \mathrm{GHz}$ ). This will lead to the generation of a FWM signal in the same frequency band, along TE

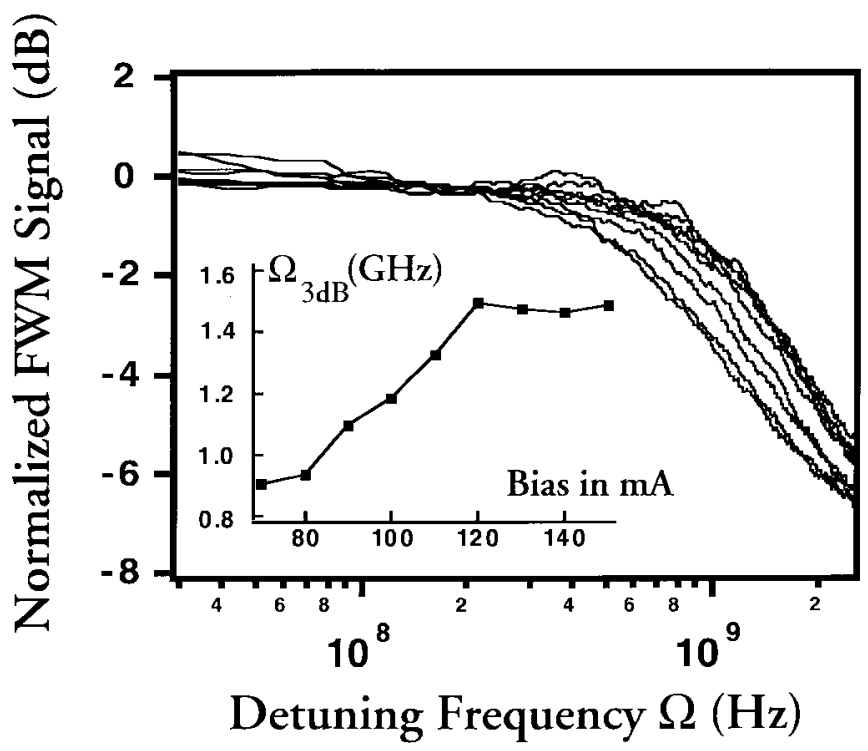

Fig. 3. Frequency dependence of the TE-polarized FWM efficiency (normalized by the TM polarization emission) as a function of detuning for SOA bias currents ranging from $50-150 \mathrm{~mA}$ (left to right). The inset shows the corresponding 3-dB roll-off frequency.

and TM. Optical filtering of the ASE noise was necessary in order to increase the gain of the second EDFA (and thus the conversion efficiency) for the narrow wavelength range of interest here. If we set the output polarization filter (PF 2) to TM, the RF-spectral power will be dominated by the amplified pump-"probe" beat noise, which is much stronger than the pump-FWM beat noise. On the other hand, if we set the output polarization filter to TE, then only the pump-FWM beat will be present, since the "probe" has no TE component. This signal component will contain the SOA carrier dynamics. In addition, of course, there will always be an almost white noise component coming from the beat between the pump and the stimulated emission of the SOA, but under highly saturated operating conditions this contribution is much weaker than the above two noise sources. The high saturation condition for this measurement limits the applicability of this technique, but is consistent with operation of cross-gain/cross-phase wavelength converters.

To remove the frequency dependence of our detection electronics, the pump-FWM signal (TE) was normalized to the pump-"probe" noise (TM). The result is shown in Fig. 3, where we can clearly identify the 3-dB corner frequency of the FWM conversion efficiency. The total input power into the device was $12.3 \mathrm{dBm}$, and the optical pump signal (laser) to ASE power ratio was $6.6 \mathrm{~dB}$ (measured into $0.1 \mathrm{~nm}$ bandwidth). The SOA was $780-\mu \mathrm{m}$ long and had three pairs of compressive/tensile strained quantum wells [8]. The proper input polarization states were selected using a polarizer at the input and by minimizing the power in the orthogonal direction. Since the TE generated RF-signal results from beating of the pump with both FWM sidebands, this is a measure of the amplitude response of the FWM at very low frequencies (i.e., contains no FWM phase information).

The use of a mixed strain amplifier for this experiment is not a prerequisite, and the method is in principle applicable to 
any SOA with optical transitions involving the light hole band (tensile-strained quantum wells or bulk amplifiers). Amplifiers consisting of alternating pairs of quantum wells, as is the case here, are slightly more complicated in that the dynamics involved in the generation of the TE polarized FWM signal are different for the two different types of wells. In the tensile wells, the TE matrix element is nonzero, and the carrier modulation in these wells translates directly into a gain and index modulation for that mode. On the other hand, in the compressive wells, the TM-induced carrier modulation must first be transferred over from the adjacent wells by means of interwell transport. We have recently characterized the dynamics associated with this process [6], and found that the modulation transfer time constant is much faster than the spontaneous lifetime measured here, which means that the results at hand are not affected by the interwell dynamics.

We applied this experimental method to map out the dependence of the stimulated lifetime on the bias current of the SOA (under conditions of fixed input power). The curves in Fig. 3 correspond to an SOA current bias ranging from 50 to 150 $\mathrm{mA}$ in steps of $10 \mathrm{~mA}$. The inset shows how the stimulated lifetime changes with current bias. Since the input power into the device is high enough to fully saturate the amplifier for all the bias conditions, this curve reflects the change of the saturated gain with current.

\section{CONCLUSION}

We have proposed and demonstrated a novel technique to characterize the stimulated carrier recombination rate in semiconductor optical amplifiers containing tensile strained quantum wells. The method takes advantage of the polarization properties of FWM to measure the pole occurring at $1 / \tau_{s}$ in the conversion efficiency versus detuning frequency.

\section{ACKNOWLEDGMENT}

The authors thank Dr. N. Kwong and Dr. T. Schrans, ORTEL, Corp., for the loan of the DFB laser and the PIN detector used in this experiment.

\section{REFERENCES}

[1] T. Durhuus, B. Mikkelsen, and C. Joergensen, "All-optical wavelength conversion by semiconductor optical amplifiers," J. Lightwave Technol., vol. 14, pp. 942-954, 1996.

[2] H. Yasaka, T.-K. Hishii, K. Oe, Y. Yoshikuni, and H. Tsuchiya, "Broadrange wavelength conversion of $10 \mathrm{~GB} / \mathrm{s}$ signal using a superstructure grating DBR laser," Electron. Lett., vol. 30, pp. 133-134, 1994.

[3] D. Marcenac, A. Kelly, D. Nesset, and D. Davis, "Bandwidth enhancement of wavelength conversion via cross-gain modulation by semiconductor optical amplifier cascade," Electron. Lett., vol. 31, pp. 1442-1443, 1995.

[4] G. P. Agrawal, "Population pulsations and nondegenerate four-wave mixing in semiconductor lasers and amplifiers," J. Opt. Soc. Amer. B, vol. 5, pp. 147-158, 1988

[5] J. Zhou, N. Park, J. W. Dawson, K. J. Vahala, M. A. Newkirk, and B I. Miller, "Efficiency of broadband four-wave mixing wavelength conversion using semiconductor traveling-wave amplifiers," IEEE Photon. Technol. Lett., vol. 6, pp. 50-52, 1994.

[6] R. Paiella, G. Hunziker, K. J. Vahala, and U. Koren, "Measurement of the interwell carrier transport lifetime in multiquantum-well optical amplifiers by polarization-resolved four-wave mixing," Appl. Phys. Lett., vol. 69, pp. 4142-4144, 1996.

[7] R. Paiella, G. Hunziker, J. Zhou, K. J. Vahala, U. Koren, and B. I. Miller, "Polarization properties of four-wave mixing in strained semiconductor optical amplifiers," IEEE Photon. Technol. Lett., vol. 8, pp. 773-775, 1996.

[8] M. A. Newkirk, B. I. Miller, U. Koren, M. G. Young, M. Chen, R. M. Jopson, and C. A. Burrus, "1.5 $\mu \mathrm{m}$ multi-quantum-well semiconductor optical amplifier with tensile and compressively strained wells for polarization-independent gain," IEEE Photon. Technol. Lett., vol. 5, pp. 406-408, 1993. 